\title{
Harmonic scalpel versus electrocautery for harvest of radial artery conduits: Reduced risk of spasm and intimal injury on optical coherence tomography
}

Philip S. Brazio, BS, BA, ${ }^{a}$ Patrick C. Laird, BA, ${ }^{a}$ Chenyang Xu, PhD, ${ }^{\mathrm{b}}$ Junyan $\mathrm{Gu}, \mathrm{MD}, \mathrm{PhD},{ }^{\mathrm{a}}$ Nicholas S. Burris, BS, ${ }^{\mathrm{a}}$ Emile N. Brown, BS, ${ }^{a}$ Zachary N. Kon, BA, ${ }^{a}$ and Robert S. Poston, MD ${ }^{c}$

Objective: Vasospasm is the primary obstacle to widespread adoption of the radial artery as a conduit in coronary artery bypass grafting. We used optical coherence tomography, a catheter-based intravascular imaging modality, to measure the degree of radial artery spasm induced by means of harvest with electrocautery or a harmonic scalpel in patients undergoing coronary artery bypass grafting.

\begin{abstract}
Methods: Radial arteries were harvested from 44 consecutive patients with a harmonic scalpel $(n=15)$ or electrocautery $(n=29)$. Vessels were imaged before harvesting and after removal from the arm, with saphenous vein tracts serving as internal controls. Optical coherence tomographic findings for the degree of harvesting-induced injury were validated against histologic measures.

Results: Optical coherence tomographic measures of endovascular dimensions and injury correlated strongly with histologic findings. Mean luminal volume, a measure of vasospasm, decreased significantly less after harvesting with a harmonic scalpel $(9 \% \pm 7 \%)$ than with electrocautery $(35 \% \pm 6 \%, P=.015)$. Completely intact intima was present in $11(73 \%)$ of 15 radial arteries harvested with a harmonic scalpel (73\%) compared with 9 of 29 arteries harvested by means of electrocautery $(31 \%, P=.011)$. Intraoperative flow measurements and patency rates at 5 days postoperatively were not significantly different among groups.

Conclusions: Optical coherence tomography provides a level of speed and accuracy for quantifying endothelial injury and vasospasm that has not been described for any other modality, suggesting potential as an intraoperative quality assurance tool. Our optical coherence tomographic findings suggest that the harmonic scalpel induces less spasm and intimal injury compared with electrocautery.
\end{abstract}

\begin{abstract}
Although the internal thoracic artery is the conduit of choice for coronary artery bypass grafting (CABG), the saphenous vein and radial artery (RA) continue to be harvested and used for patients who require multiple grafts. ${ }^{1}$ A recent randomized trial has shown that RA grafts retain better patency 1 year after revascularization compared with saphenous vein grafts (SVGs). ${ }^{2}$ In this same trial, however, 7\% of RA grafts had diffuse spasm, as evidenced by means of radiographic "string sign" and suboptimal or Thrombolysis In Myocardial Infarction grade 1 blood flow. ${ }^{3}$ Although the SVG is limited by acute thrombosis and neointimal hyperplasia, spasm is the predominant factor preventing the routine adoption of the RA as a conduit in CABG.

The risk of RA spasm relates to several factors, one of which is the degree of stenosis within the native vessel that is being

From the Division of Cardiac Surgery, University of Maryland School of Medicine, ${ }^{\mathrm{a}}$ Baltimore, Md; LightLab Imaging, ${ }^{\mathrm{b}}$ Westford, Mass; and Division of Cardiac Surgery, Boston University Medical School, ${ }^{\mathrm{c}}$ Boston, Mass.

Dr Poston is supported by grants from the National Institutes Health (RO1 HL080410) and the American Heart Association (Scientist Development Grant). Dr Xu is an employee of LightLab Imaging, the manufacturer of the optical coherence tomographic device used in this study.

Received for publication March 8, 2008; revisions received April 27, 2008; accepted for publication May 25, 2008.

Address for reprints: Robert S. Poston, MD, Boston Medical Center, Division of Cardiac Surgery, Robinson 402, 88 E. Newton Street, Boston, MA 02118 (E-mail: robert.poston@bmc.org).

J Thorac Cardiovasc Surg 2008;136:1302-8

$0022-5223 / \$ 34.00$

Copyright (c) 2008 by The American Association for Thoracic Surgery doi: $10.1016 /$ j.jtcvs. 2008.05 .060
\end{abstract}

grafted. It is well established that placing the RA onto a target coronary vessel that has less than $70 \%$ stenosis creates competitive flow and is associated with a higher risk of RA spasm compared with target arteries with greater degrees of stenosis. ${ }^{4}$ Additional evidence implicates risk factors at the level of the graft itself. Comorbid conditions linked to endothelial disease, such as diabetes and peripheral atherosclerosis, have been found to predispose this conduit to vasospasm. ${ }^{5-8}$ An important and modifiable risk factor that influences the status of the RA endothelium is the method used to harvest the vessel. Improvements in techniques have been instrumental in maintaining endothelial integrity and returning the RA to popularity as a conduit for CABG in the 1990s. ${ }^{9}$

One harvesting technique that has been purported to reduce spasm of the RA graft is the use of a harmonic scalpel instead of electrocautery with hemoclips. ${ }^{10}$ Although it has been suggested that this technique improves endothelial integrity, other studies have failed to confirm this finding. Verifying the optimal technique for RA harvest would be greatly aided by an accurate, objective, reproducible method for detecting spasm and intimal integrity within this conduit. At present, the only widely used objective measure of vasospasm is catheter-based angiography. This technique is not feasible for intraoperative use and has suboptimal resolution for quantifying RA spasm or injury.

Optical coherence tomography (OCT) is a novel imaging modality that measures the reflection of emitted infrared 


\section{Abbreviations and Acronyms \\ $\mathrm{CABG}=$ coronary artery bypass grafting \\ CTA = computed tomographic angiography \\ OCT = optical coherence tomography \\ $\mathrm{RA}=$ radial artery \\ $\mathrm{SVG}=$ saphenous vein graft}

radiation and is particularly suited for imaging the luminal surface of blood vessels. We have demonstrated that OCT images obtained during the course of $\mathrm{CABG}$ provide clinically meaningful information in real time about the quality of harvested bypass conduits. ${ }^{11,12}$ In addition, image-processing software used with OCT can detect the luminal boundaries of the RA as a means of quantifying spasm. The purpose of this study was to assess the incidence of vasospasm and endothelial injury, as defined by OCT and validated against histologic measures, among patients undergoing $\mathrm{CABG}$ with a harmonic scalpel or electrocautery.

\section{MATERIALS AND METHODS \\ Patient Population}

Consecutive patients were eligible if they were considered for an RA conduit during isolated CABG between March 2006 and April 2007 at our institution. Exclusion criteria included a serum creatinine level of greater than $2.0 \mathrm{mg} / \mathrm{dL}$, refusal or inability to provide informed consent, an abnormal oximetric Allen's test result, hemodialysis requirement, uncontrolled diabetes $\left(\mathrm{HgAl}>\mathrm{c}>\mathrm{\%}\right.$ ), or Raynaud's disease. ${ }^{13}$ The protocol (no. H25350) was approved by the University of Maryland, Baltimore institutional review board, and all patients provided written informed consent.

\section{Surgical Technique}

RAs were harvested by a single technician (KS) with either a harmonic scalpel (Ethicon, Somerville, NJ) using an open incision technique or the combination of electrocautery and clips for the vessel branches. Group assignment was nonrandomized. All patients also had SVGs harvested endoscopically and evaluated by means of OCT as an internal control for comparison of vessel friability versus the RA. ${ }^{14}$ After removal of the RA from the forearm and before re-examination by means of OCT, the conduit was flushed with heparinized saline and stored in a Plasmalyte (Baxter Healthcare Corp., Deerfield, Ill.) solution containing glyceryl trinitrate and verapamil. ${ }^{15}$

A single surgeon (RP) carried out off-pump CABG through a median sternotomy, with distal anastomoses performed with the use of suctionbased exposure and stabilizing devices (Octopus 4.3; Medtronic, Inc, Minneapolis, Minn). Heparin was administered at the completion of harvesting at a dose calculated to obtain an activated clotting time of longer than 300 seconds and reversed by half the dose of protamine calculated by means of heparin-protamine titration. Aspirin $(325 \mathrm{mg}$ ) was administered orally before the procedure and continued after the operation.

\section{OCT Imaging}

The RA was first imaged in situ by means of insertion of an OCT catheter (ImageWire; LightLab Imaging, Westford, Mass) into the forearm (Figure 1, A) by using an upper-arm tourniquet and infusion of heparinized saline to clear blood. The catheter was then withdrawn at $1.0 \mathrm{~mm} / \mathrm{s}$ by using an automated pullback device, yielding sequential transverse views of the vessel with a frame rate of $10 \mathrm{~Hz}$ and a slice width of $0.1 \mathrm{~mm}$. The RA was exam- ined again after harvesting and removal from the limb (Figure 1, B), as was the harvested tract of saphenous vein.

OCT images were analyzed by 2 different technicians (PL and PB) who were unaware of the harvesting technique. Harvesting injury was categorized as minor when intimal disruption was restricted to the ostia of branch points or severe if it involved the luminal surface ${ }^{16}$ The luminal volume of the RA was calculated from OCT images by using automated imaging-processing software; perioperative change was quantified by comparing the luminal volume before and after harvesting.

\section{Biopsy Confirmation of OCT Findings}

Biopsy specimens were obtained from specific regions of interest within the discarded segment of RAs. Regions of vascular trauma visualized by means of OCT imaging were marked externally on the vessel at the location of the catheter tip and visualized by means of gross examination of a rotating infrared light (Figure 1, $B$, inset). These "image-guided" biopsy sections were embedded and frozen in cutting compound (Tissue-Tek O.C.T.; Ted Pella, Inc, Redding, Calif) and sectioned at $5 \mu \mathrm{m}$. Sections were stained for elastin by using a kit (SciTec Laboratories, Logan, Utah) and endothelial integrity, as previously described.$^{17}$ Luminal tissue factor activity was determined in vessel biopsy specimens isolated in a custom-designed chamber. A reaction buffer was added containing Factors VIIa (2 U/mL; American Diagnostica, Greenwich, Conn) and X (2 U/mL, American Diagnostica), with ethylenediamine tetraacetic acid used to stop the reaction. The generation of Factor Xa was measured in the supernatant by adding a chromogenic substrate (Spectrozyme FXa, American Diagnostica), and the absorption was measured at $405 \mathrm{~nm}$.

\section{Graft Patency Analysis}

Blood flow was measured in each RA graft intraoperatively by using transit-time ultrasonography (VeriQ; Medi-Stim, Inc, Oslo, Norway). Measurements were obtained before and after native artery occlusion to rule out competitive flow. ${ }^{18}$ Graft patency at 1 week was determined by means of blinded review (CW) of computed tomographic angiographic (CTA) images (420-ms rotation, $100-150 \mathrm{~mL}$ of contrast agent administered intravenously at $5 \mathrm{~mL} / \mathrm{s}$ ) with retrospective electrocardiographic gating. ${ }^{19}$ Patency was defined as FitzGibbon grade A (ie, luminal diameter of the RA conduit exceeding that of its coronary target through the entire length of the graft). ${ }^{20}$

\section{Statistical Analysis}

The primary end point of this study was the degree of intraoperative vasospasm and intimal injury in RA conduits harvested with a harmonic scalpel versus electrocautery. Previous use of OCT as a quality assurance tool demonstrated a 5 -fold difference in intimal injury between open and endoscopic methods for RA conduit harvesting. ${ }^{11}$ We assumed a more conservative $50 \%$ relative difference in the incidence of intimal injury between the 2 groups in this study. Therefore 25 patients per group were expected to provide $80 \%$ power to detect this difference at a $P$ value of .05 in 2 -tailed testing. Spasm, defined as the percentage change in mean luminal volume between the in situ and ex vivo OCT scans, was compared by means of the $t$ test. The reproducibility of our OCT interpretations was quantified by using interobserver $\kappa$ correlation coefficients.

The relation between demographic and clinical patient characteristics and the degree of RA spasm was assessed by means of univariate regression analysis (for continuous variables) and the $t$ test (for categorical variables). Statistical analysis was performed with the InStat statistical package (version 3.0; GraphPad Software, Inc, San Diego, Calif) with assistance of a biostatistician (AJ).

\section{RESULTS}

\section{Study Population}

OCT examinations were performed on a total of 44 patients, with the harmonic scalpel used for 15 harvests and 

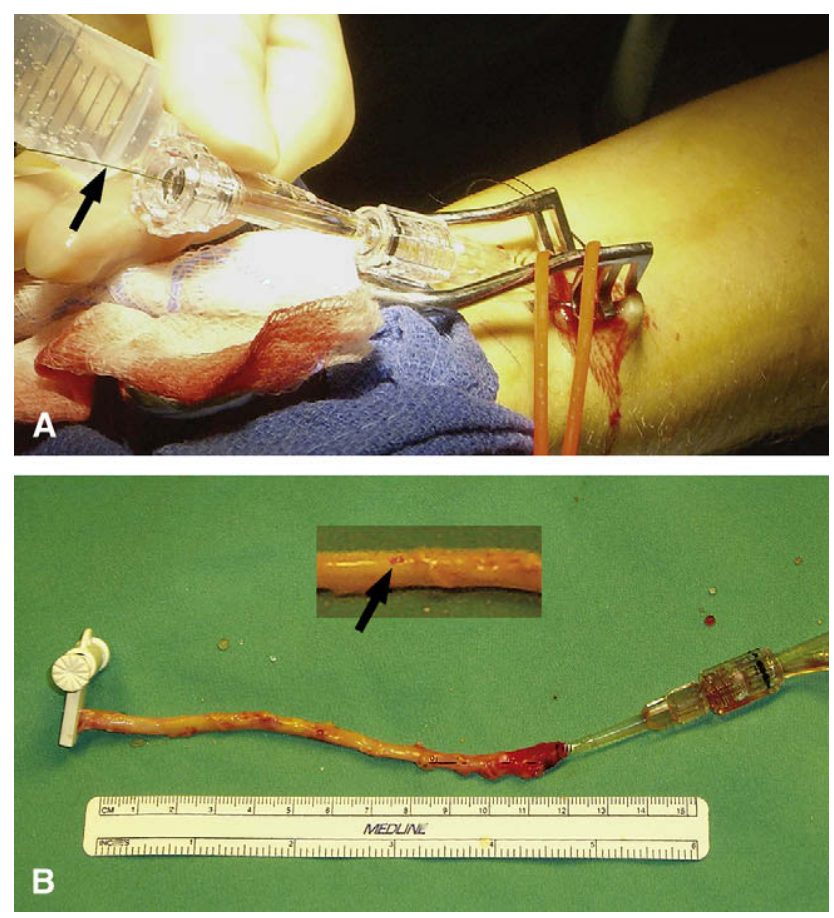

FIGURE 1. Localization of the optical coherence tomographic imaging probe within the radial artery. The infrared light emitted from the tip of the optical coherence tomographic probe (A) can easily be visualized through the luminal wall of the radial artery (arrow, B) during image acquisition. This enables external marking of abnormal areas of the artery, thereby providing a roadmap for selecting the optimal portion of the conduit for bypass. Imaging-guided biopsy specimens also can be obtained for histologic confirmation of optical coherence tomographic findings.

electrocautery for 29 harvests within an average examination time of $2.7 \pm 0.3$ minutes per conduit. Baseline characteristics and comorbid conditions were similar between the 2 groups, except there were significantly more patients with peripheral vascular disease in the harmonic group (Table 1). Predischarge CTA was performed in all but 3 patients because of a serum creatinine level of greater than $2.0 \mathrm{mg} / \mathrm{dL}$.

\section{Validation of OCT Findings}

Analysis of registered biopsy sections demonstrated that endothelial integrity, tissue factor, and disruption to the internal elastic lamina, as defined by elastin staining, differed significantly between portions of RA diagnosed by means of imaging as normal versus severely injured (Figure 2). Identification of intimal damage with OCT was highly reproducible, as evidenced by interobserver $\kappa$ correlation values of 0.85 for minor trauma and 1.0 for severe trauma. A subset of RAs in this study underwent targeted biopsy of abnormal areas detected by means of OCT imaging to obtain further histologic and biochemical analyses (Table 2). Areas with severe intimal injury per OCT had significantly higher tissue factor activity, lower endothelial integrity (as measured by means of CD31 staining), and a higher number of intimal
TABLE 1. Baseline demographic and clinical characteristics

\begin{tabular}{lccc}
\hline & $\begin{array}{c}\text { Harmonic scalpel Electrocautery } \\
(\mathbf{n}=\mathbf{1 5})\end{array}$ & $\begin{array}{c}\text { (n = 29) } \\
\text { P value }\end{array}$ & $\boldsymbol{P}$ \\
\hline Age $(\mathrm{y})$ & 66.5 & 66.7 & .949 \\
Body mass index $\left(\mathrm{kg} / \mathrm{m}^{2}\right)$ & 28.3 & 29.1 & .683 \\
Male sex & 86.7 & 96.0 & .714 \\
Active smoking & 33.3 & 29.2 & .791 \\
Family history of coronary & 58.3 & 40.0 & .308 \\
$\quad$ disease & & & \\
Hypertension & 92.9 & 92.0 & .926 \\
Hyperlipidemia & 92.3 & 82.6 & .433 \\
Carotid artery disease & 21.4 & 20.0 & .918 \\
Previous stroke & 14.3 & 8.0 & .547 \\
Peripheral vascular disease & 38.5 & 8.0 & .021 \\
Diabetes mellitus & 50.0 & 47.8 & .901 \\
CCS class >2 angina & 80.0 & 79.3 & .958 \\
NYHA class >II heart failure & 86.7 & 86.2 & .967 \\
\hline
\end{tabular}

Data are expressed as the mean value or percentage of patients. CCS, Canadian Cardiovascular Society; NYHA, New York Heart Association.

tears on elastin staining compared with areas with no intimal injury per OCT.

\section{Intraoperative Vasospasm}

The luminal volume of the RA, as determined by means of OCT imaging, was similar between groups at baseline. Reassessment of RA luminal volume after harvest showed a significantly greater decrease after the use of electrocautery compared with harmonic scalpel (Table 3). Longitudinal plots reconstructed with image-processing software illustrated that spasm was mainly diffuse, rather than focal (Figure 3). Other than the technique used for harvest, the clinical variables analyzed showed no correlation to the decrease in RA luminal volume.

\section{Intimal Injury}

Endothelial integrity measured by means of CD31 staining of random biopsy specimens obtained from each RA did not show differences between harvesting techniques $(52 \% \pm 30 \%$ for the harmonic scalpel vs $42 \% \pm 28 \%$ for electrocautery, $P=.65)$. On the other hand, OCT examination demonstrated that $11(73 \%)$ of 15 RAs harvested with a harmonic scalpel had a completely normal intimal layer, with no evidence on OCT imaging of focal intimal trauma, compared with $9(31 \%)$ of 29 arteries harvested by means of electrocautery $(P=.011)$. Most intimal defects $(63 \%)$ were confined to the ostium of branch points. Tears involving the luminal surface were found in $20 \%$ of the harmonic scalpel samples and $45 \%$ of the electrocautery samples $(P=.186)$, whereas minor (ostial) tears were found in $13 \%$ of harmonic scalpel samples and $45 \%$ of electrocautery samples $(P=.048)$. In contrast, control SVG segments harvested with electrocautery showed no severe injury, and ostial tears were rare, found in only $26(2.9 \%)$ of 884 assessed branch points. 

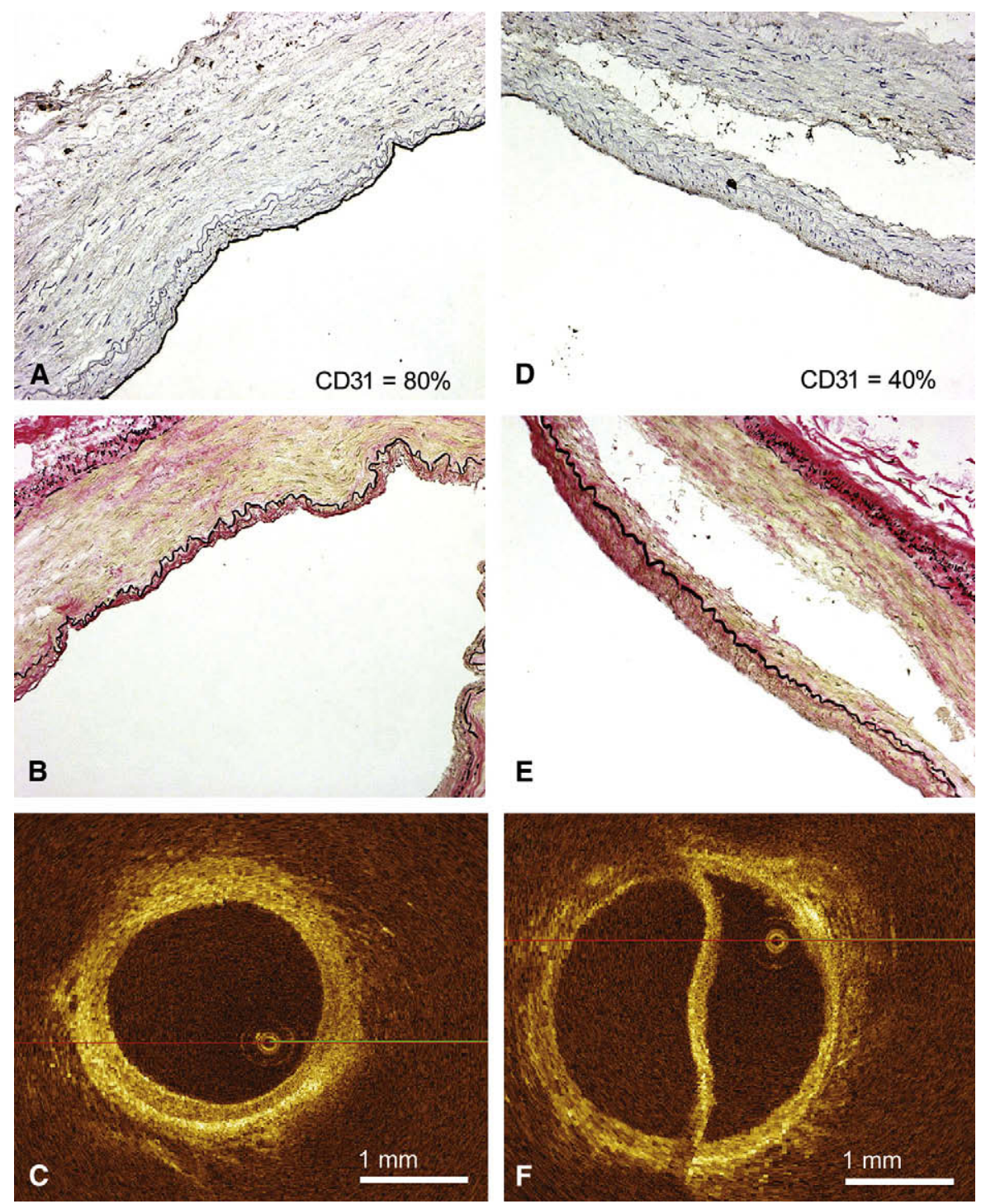

E
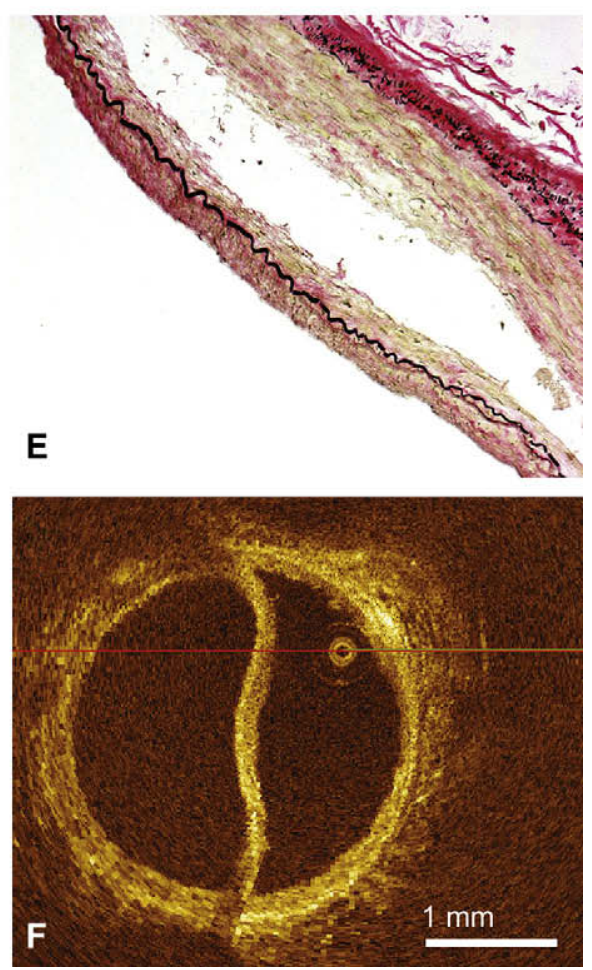

FIGURE 2. Endothelial integrity of harvested radial artery portions according to histologic findings (CD31 staining, A and D; elastin staining, B and E) and optical coherence tomographic analysis (C and F). A, B, and C, Normal artery portions; D, E, and F, artery portions with dissection.

\section{Postoperative Vasospasm and Patency}

After grafting, RAs harvested with a harmonic scalpel versus electrocautery showed no differences in blood flow measured intraoperatively $(43.8 \pm 5.8 \mathrm{~mL} / \mathrm{min}$ for harmonic scalpel vs $44.0 \pm 5.0 \mathrm{~mL} / \mathrm{min}$ for electrocautery, $P=.98$ ) but a trend toward an increased pulsatility index (3.12 vs 2.26, $P=.05$ ). CTA on day 5 showed 14 of 15 grafts from the harmonic scalpel group and 24 of 29 grafts from the electrocautery group with grade A patency $(P=.24)$.

\section{DISCUSSION}

We describe the novel use of a high-resolution imaging technique called OCT to demonstrate that surgical harvest with a harmonic scalpel yields RA conduits of higher quality than provided by the standard technique used at most CABG centers, electrocautery and clips. Although the influence of competitive flow and choice of coronary target on RA patency have been thoroughly established, the potential for the intimal quality of the RA to influence patency has been underappreciated. OCT images of the RA obtained after harmonic scalpel procurement revealed significantly less intimal defects and change in luminal volume, suggesting less trauma-induced endothelial disruption and spasm. Because images were obtained from each conduit before and after procurement, the most practical explanation for our findings is that the harmonic scalpel provides a more meticulous technique for handling the RA. Given evidence that the RA is more friable than the SVG, the harmonic scalpel might overcome some of the perceived drawbacks of this controversial conduit. $^{21}$ 
TABLE 2. Comparison of OCT-guided biopsy specimens versus biopsy-based evidence of conduit injury

\begin{tabular}{lccc}
\hline & $\begin{array}{c}\text { Severe intimal } \\
\text { injury per } \\
\text { OCT }(\mathbf{n}=\mathbf{5})\end{array}$ & $\begin{array}{c}\text { No intimal } \\
\text { injury per } \\
\text { OCT }(\mathbf{n}=\mathbf{1 0})\end{array}$ & $\boldsymbol{P}$ value \\
\hline $\begin{array}{c}\text { Tissue factor activity (U) } \\
\text { CD31 expression (\% of } \\
\quad \text { luminal circumference) }\end{array}$ & $5.3 \pm 1.4$ & $1.4 \pm 1.0$ & .01 \\
$\begin{array}{c}\text { IEL disruption per elastin } \\
\text { staining (no. of tears) }\end{array}$ & $6.0 \pm 3.3$ & $64 \pm 20$ & .01 \\
& & $0.5 \pm 1.4$ & .05 \\
\hline
\end{tabular}

staining (no. of tears)

Data are expressed as mean values \pm standard deviation. OCT, Optical coherence tomography; $I E L$, internal elastic lamina.

Our results conflict with a recent meta-analysis of randomized controlled trials comparing these techniques, which suggested that evidence in favor of the harmonic scalpel is "not convincing.", 22 Despite far less enrollment in our study, 44 versus 349 collective patients in the meta-analysis, ${ }^{23-28}$ OCT imaging dramatically improves the ability to detect subtle changes in conduit quality. Prior assessments of RA spasm or injury during harvesting have been limited to either visual examination/palpation ${ }^{27}$ or ex vivo assays performed on surplus portions of the RA. ${ }^{23,27}$ Luminal

TABLE 3. Measures of intraoperative vasospasm by harvesting method

\begin{tabular}{lccc}
\hline & $\begin{array}{c}\text { Harmonic scalpel } \\
(\mathbf{n}=\mathbf{1 5})\end{array}$ & $\begin{array}{c}\text { Electrocautery } \\
(\mathbf{n}=\mathbf{2 9})\end{array}$ & $\boldsymbol{P}$ value \\
\hline Luminal volume & & & \\
$\quad$ Baseline $\left(\mathrm{mm}^{3}\right)$ & $1079 \pm 112$ & $906 \pm 86$ & .237 \\
After harvesting $\left(\mathrm{mm}^{3}\right)$ & $921 \pm 80^{*}$ & $559 \pm 69 \dagger$ & .003 \\
$\begin{array}{l}\text { Percentage change } \\
\quad \text { between scans }\end{array}$ & $8.8 \pm 7.1$ & $34.5 \pm 6.2$ & .015 \\
\hline $\begin{array}{l}\text { Data are expressed as mean values } \pm \text { standard error. }{ }^{*} P=.87 \text { versus baseline. } \\
\dagger P=.0002 \text { versus baseline. }\end{array}$
\end{tabular}

spasm, intimal tears, or both in harvested RAs occur heterogeneously within the vessel and are rarely associated with detectable abnormalities based on the gross external appearance. ${ }^{17}$ Histology, electronic microscopy, and physiologic studies performed on rings of vascular tissue are sensitive measures of RA integrity but are limited by sampling bias and are not able to assess the grafted portion of the RA. The reliability of intravascular ultrasonic screening to identify intimal pathology is uncertain. ${ }^{12}$ With an axial resolution in the range of 2 to $15 \mu \mathrm{m},{ }^{12}$ OCT is a far more suitable technique for identifying pathology, such as intimal
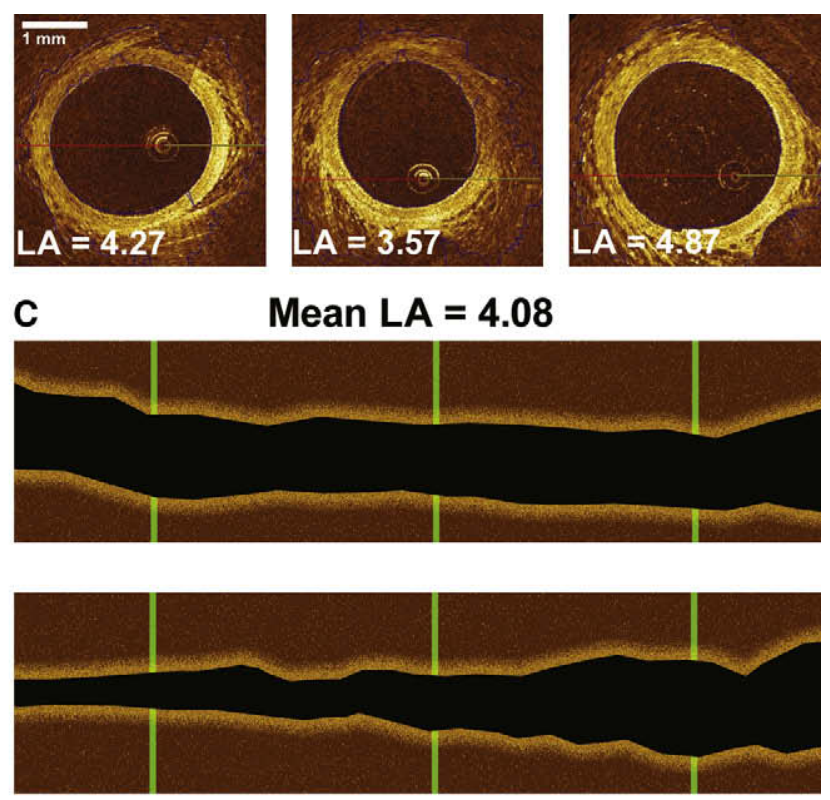

D

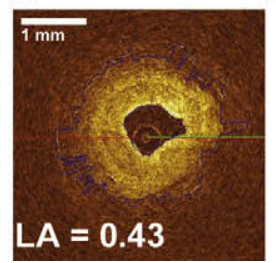

Mean LA $=1.35$

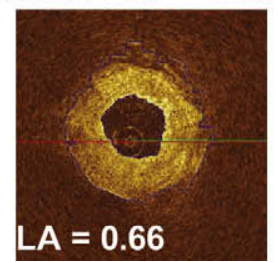

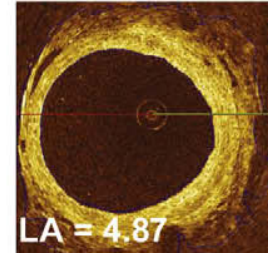

FIGURE 3. Optical coherence tomographic frames at 3 points of the radial artery before (A and C) and after (B and D) harvest. The mean luminal area $(L A)$ of the artery harvested with the harmonic scalpel (A and B) increased by $6.5 \%$, whereas the LA of the artery harvested by means of electrocautery decreased by $66.9 \%$ (C and D). After harvesting, panel B shows small areas of mild focal spasm, whereas panel D displays severe diffuse spasm (string sign). 
trauma, anywhere within the RA and for quantifying the degree of intraoperative spasm after harvest.

The current analysis adds to the growing evidence supporting the value of OCT as a tool for assessing the quality of bypass conduits used for CABG. Previously, we noted that endoscopic harvest of SVGs yields minimal adverse effect on intimal quality but results in an alarming incidence of retained clot compared with open harvest. ${ }^{29}$ The availability of OCT images in real time greatly enhances the relevance of this information to the surgical team. For example, instant demonstration of how an early dose of heparin eliminates clot retention within the SVG improves compliance with this therapy by placing adverse effects on the risk of bleeding in better context. Evidence of intimal injury or spasm in the conduit provides valuable feedback to surgical technicians early in their learning curve.

\section{Implications and Limitations for Clinical Practice}

The ability to note which sections of conduit contain severe pathology (eg, focal plaques or intimal dissections) in the operating room allows that portion to be excluded from the conduit that is selected for grafting. As shown with registered vessel biopsy specimens, this intervention helps to avoid areas of disrupted endothelium and increased tissue factor and thereby restore the anticoagulant and antiinflammatory properties that characterize a normal conduit. The central role of the intima in graft performance is supported by the stark contrast in patency rates for the internal thoracic artery versus the SVG, as well as our previous demonstration that the proportion of denuded surface area within a harvested SVG correlates with reduced patency. ${ }^{30}$

As with any new technology, OCT imposes an expense. The cost of each disposable probe (approximately $\$ 300$ $\$ 400$ ) must be accounted for. A dedicated technician is required to help carry out the intraoperative conduit evaluation. Qualitative assessment of spasm from OCT imaging in real time, including setup and scanning, can add 10 to 20 minutes of time to a case. More streamlined iterations of the current software that are available for these analyses will reduce procedural times in the future.

\section{Study Limitations}

This study was not a randomized comparison of harvesting methods, and thus the possibility of bias exists. We attempted to minimize this possibility in several ways. First, we included consecutive patients in the study, rather than targeting certain patients for a given harvesting method. Second, abnormalities on OCT were confirmed in each case by histologic findings performed by reviewers who were unaware of the harvesting method. Third, we assessed the incidence of spasm according to baseline clinical and demographic characteristics, as well as graft patency, to identify variables that might have confounded the analysis.
Another limitation was that our study was not appropriately powered to confirm that conduit abnormalities detected by means of OCT anticipate problems with early patency and showed a nonsignificant difference between groups in early patency or intraoperative flow rate. On occasion, OCT imaging has revealed severe intimal injury after harvest that is consistent with a vessel dissection. For extreme cases such as this, we believe that it is obvious that the conduit should not be used. However, our experience with screening of conduits is in its early stages. We cannot at this point establish whether more subtle findings detected on OCT imaging put the RA conduit at higher risk of early failure. Our data should be considered hypothesis generating until confirmed by a randomized controlled trial of sufficient power to detect significant differences between outcomes with these 2 harvesting methods.

\section{CONCLUSIONS}

Although this study was small, the use of OCT imaging provided a rapid and accurate means of quantifying RA spasm and endothelial injury. When applied as an intraoperative quality assurance tool in patients undergoing CABG, OCT demonstrated that use of a harmonic scalpel was associated with significantly less RA spasm and intimal injury versus electrocautery. Future trials comparing novel methods for conduit harvesting should consider incorporating this innovative technology into their study protocol.

\section{References}

1. American College of Cardiology/American Heart Association Task Force on Practice Guidelines (Committee to Update the 1999 Guidelines for Coronary Artery Bypass Graft Surgery). ACC/AHA 2004 guideline update for coronary artery bypass graft surgery (erratum appears in Circulation. 2005;111:2014). Circulation. 2004;110:e340-437.

2. Desai ND, Cohen EA, Naylor CD. Fremes SE, for the Radial Artery Patency Study Investigators. A randomized comparison of radial-artery and saphenousvein coronary bypass grafts. $N$ Engl J Med. 2004;351:2302-9.

3. Miwa S, Desai N, Koyama T, et al. Radial artery angiographic string sign: clinical consequences and the role of pharmacologic therapy. Ann Thorac Surg. 2006;81: 112-8.

4. Chong CF, Moat NE, Collins P. Radial artery grafts' string-sign-role of graft spasm and competitive flow. Interact Cardiovasc Thorac Surg. Epub September $15,2006$.

5. Desai ND, Naylor CD, Kiss A, et al. Impact of patient and target-vessel characteristics on arterial and venous bypass graft patency: insight from a randomized trial. Circulation. 2007;115:684-91.

6. Gaudino M, Nasso G, Canosa C, et al. Midterm angiographic patency and vasoreactive profile of proximal versus distal radial artery grafts. Ann Thorac Surg. 2005;79:1987-9

7. Kim YO, Song HC, Yoon SA, et al. Preexisting intimal hyperplasia of radial artery is associated with early failure of radiocephalic arteriovenous fistula in hemodialysis patients. Am J Kidney Dis. 2003;41:422-8.

8. Choudhary BP, Antoniades C, Brading AF, Galione A, Channon K, Taggart DP. Diabetes mellitus as a predictor for radial artery vasoreactivity in patients undergoing coronary artery bypass grafting. J Am Coll Cardiol. 2007;50:1047-53.

9. Acar C, Jebara VA, Portoghese M, et al. Revival of the radial artery for coronary artery bypass grafting. Ann Thorac Surg. 1992;54:652-9.

10. Oz BS, Mataraci I, Iyem H, et al. Comparison of ultrasonically activated scalpel and traditional technique in radial artery harvesting: clinical research. Thorac Cardiovasc Surg. 2007;55:104-7. 
11. Burris N, Schwartz K, Tang CM, et al. Catheter-based infrared light scanner as a tool to assess conduit quality in coronary artery bypass surgery. $J$ Thorac Cardiovasc Surg. 2007;133:419-27.

12. Burris NS, Brown EN, Grant M, et al. Optical coherence tomography imaging as a quality assurance tool for evaluating endoscopic harvest of the radial artery. Ann Thorac Surg. 2008;85:1271-7.

13. Barbeau GR, Arsenault F, Dugas L, Simard S, Lariviere MM. Evaluation of the ulnopalmar arterial arches with pulse oximetry and plethysmography: comparison with the Allen's test in 1010 patients. Am Heart J. 2004;147:489-93.

14. Muluk S, Vorp DA, Severyn DA, Gleixner S, Johnson PC, Webster MW Enhancement of tissue factor expression in veins exposed to systemic arterial pressure. J Vasc Surg. 1998;27:571-7.

15. He GW, Rosenfeldt FL, Angus JA. Pharmacological relaxation of the saphenous vein during harvesting for coronary artery bypass grafting. Ann Thorac Surg. 1993;55:1210-7.

16. Tran R, Brown EN, Burris NS. Targeted conduit selection using optical coherence tomography in coronary artery bypass grafting. Heart Surg Forum Rev. 2007;5: $7-10$.

17. Tearney GJ, Yabushita H, Houser SL, et al. Quantification of macrophage content in atherosclerotic plaques by optical coherence tomography. Circulation. 2003; 107:113-9.

18. Bolotin G, Kypson A, Nifong LW, Chitwood R. A technique for evaluating competitive flow for intraoperative decision making in coronary artery surgery. Ann Thorac Surg. 2003;76:2118-20.

19. Frazier AA, Qureshi F, Read KM, Gilkeson RC, Poston RS, White CS. Coronary artery bypass grafts: assessment with multidetector CT in the early and late postoperative settings. Radiographics. 2005;25:881-96.

20. FitzGibbon GM, Kafka HP, Leach AJ, Keon WJ, Hooper GD, Burton JR. Coronary bypass graft fate and patient outcome: angiographic follow-up of 5,065 grafts related to survival and reoperation in 1,388 patients during 25 years. $J$ Am Coll Cardiol. 1996;28:616-26.
21. Ronan JW, Perry LA, Barner HB. Sundt TM III. Radial artery harvest: comparison of ultrasonic dissection with standard technique. Ann Thorac Surg. 2000;69: 113-4.

22. Patel A, Asopa S, Dunning J. Does radial artery harvest with a harmonic scalpel result in fewer complications than standard electrocautery methods? Interact Cardiovasc Thorac Surg. 2006;5:36-41.

23. Cikirikcioglu M, Yasa M, Kerry Z, et al. The effects of the Harmonic Scalpel on the vasoreactivity and endothelial integrity of the radial artery: a comparison of two different techniques. J Thorac Cardiovasc Surg. 2001;122:624-6.

24. Wright CB, Barner HB, Gao A, et al. The advantages of the Harmonic Scalpel for the harvesting of radial arteries for coronary artery bypass. Heart Surg Forum. 2001;4:226-9.

25. Rukosujew A, Reichelt R, Fabricius AM, et al. Skeletonization versus pedicle preparation of the radial artery with and without the ultrasonic scalpel. Ann Thorac Surg. 2004;77:120-5.

26. Hata M, Shiono M, Sezai A, et al. Determining the best procedure for radial artery harvest: prospective randomized trial for early postharvest complications. J Thorac Cardiovasc Surg. 2005;129:885-9.

27. Georghiou GP, Stamler A, Berman M, Sharoni E, Vidne BA, Sahar G. Advantages of the ultrasonic harmonic scalpel for radial artery harvesting. Asian Cardiovasc Thorac Ann. 2005;13:58-60.

28. Shapira OM, Eskenazi BR, Anter E, et al. Endoscopic versus conventional radial artery harvest for coronary artery bypass grafting: functional and histologic assessment of the conduit. J Thorac Cardiovasc Surg. 2006;131: 388-94.

29. Brown EN, Kon ZN, Tran R, et al. Strategies to reduce intraluminal clot formation in endoscopically harvested saphenous veins. J Thorac Cardiovasc Surg. 2007; 134:1259-65.

30. Manchio JV, Gu J, Romar L, et al. Disruption of graft endothelium correlates with early failure after off-pump coronary artery bypass surgery. Ann Thorac Surg. 2005;79:1991-8. 